\title{
An open-source synesthesia consistency test for use on the Qualtrics platform.
}

\author{
Nicholas Root \\ University of Amsterdam
}

Synesthesia is a neurological phenomenon in which healthy individuals experience additional, automatic, and consistent perceptions unrelated to veridical sensory input. For most of the most common forms of synesthesia, the additional sensation is a color: grapheme-color synesthetes experience specific letters of the alphabet as having specific colors, tone-color synesthetes experience specific sounds as having specific colors, etc. The "gold standard" method to diagnose $\mathrm{X}$-to-color synesthesia is to measure test-retest consistency: synesthetes use a colorpicker to choose the color they experience for a particular stimulus, and then are retested minutes, months, or even years later; genuine synesthetes experience highly consistent associations across time. There is not currently an open source tool to collect color associations from synesthetes. In addition to presenting a technical barrier for entry into synesthesia research, the lack of an open source standard has also led to a proliferation of (slightly) different choices in methodology from lab to lab. In the present work, I use colorpicker experiments in synesthetes and controls to demonstrate that even small methodological details in test-retest experiments can profoundly influence the resulting data. I use this data to generate an online color picker with ideal experimental properties. The colorpicker is open source (available on a Github repository) and can be implemented by anyone with an account on the Qualtrics platform, one of the most common online experimental platforms in the world. 


\section{Introduction}

Synesthesia is a neurological phenomenon in which healthy individuals experience additional, automatic, and consistent perceptions unrelated to veridical sensory input. For example, grapheme-color synesthetes experience graphemes (e.g., letters of the alphabet) as having a consistent color ("J is dark purple"). Although there are hundreds of types of synesthesia (for a list, see http://www.daysyn.com/synesthesia-list.html), the vast majority of synesthetic experiences are of color: synesthesia of the type "X-to-color" is quite common, whereas other types of synesthesia are quite rare (Simner et al., 2006).

Until relatively recently, synesthesia's status as a genuine perceptional phenomenon was debated (Blake et al., 2005). A key advance in the study of synesthesia was the development of the test-retest consistency measure of synesthesia: synesthetes are asked to describe their experiences, are given a surprise retest, and their consistency over time is quantified. For example, grapheme-color synesthetes will easily choose the same color for a particular grapheme across months or even years, whereas controls find such a task to be nearly impossible, because they must try to use mnemonic strategies rather than simply reporting conscious perception (Asher et al., 2006). Test-retest consistency is now considered the "gold standard" diagnostic tool for synesthesia, and few studies of synesthesia are published that do not include a test-retest measure.

As the most common type of synesthesias involve color, the most common test-retest batteries involve the use of color pickers: synesthetes are presented a stimulus (e.g., a grapheme) and must use a color picker to indicate which synesthetic color they experience. This data is not only useful for measuring test-retest consistency. Using color pickers to measure synesthetic associations also enables researchers to link patterns of associations to underlying properties that influence which stimulus gets which color. For example, grapheme-color associations are influenced by numerous factors including semantic associations, letter frequency, and visual shape (see Simner, 2013, for a review). It is also necessary to collect color associations for any experiments which require customizing stimuli to each synesthete: for example, Stroop-type experiments (e.g., Kadosh et al., 2005) rely on precise measurement of synesthetic associations.

For many years, the Eagleman Synesthesia Battery (Eagleman et al., 2007) was the most widelyused tool to measure test-retest consistency. Critically, participants could opt to share their results over email, allowing researchers around the world to use the website for their own experiments (while implicitly sharing all data with the site's creators). Unfortunately, the Synesthesia Battery is now offline. In addition, the creators of the battery made several methodological choices about the color picker itself, that I will argue are suboptimal.

First, the Eagleman Synesthesia Battery - rightly - randomizes the starting position of the color picker when it is presented to the participant. This prevents the default choice from influencing participants' responses, and is critical when researchers want to measure color associations in non-synesthetes who are forced to choose colors for stimuli. However, the Eagleman Battery did not record the initial color on each trial (or, at least, this data was not accessible to external 
researchers). As a result, it was not possible to determine whether a participant interacted with the color picker at all - a "lazy" participant could simply choose the (random) initial color of the color picker on each trial. Ideally, these trials would be removed from analysis, but it was not possible to do so.

Second, the Eagleman Battery allowed participants to indicate that they experienced "no color" for a grapheme, at which point the trial would be skipped. It is important for synesthetes to be able to indicate that they do not experience a color for a particular grapheme, because this is a genuine phenomenon - for example, English letter-color synesthetes do not always experience colors for all 26 letters. However, control participants (non-synesthetes) who take the battery must be instructed not to choose "no color"; otherwise, they would logically choose this option for every trial! Therefore, comparisons of synesthetes' and non-synesthetes' performance on the battery is confounded by the fact that "no color" responses are much more meaningful for synesthetes. Furthermore, it is an open question whether synesthetes' no color responses indicate that they do not experience a consistent color; it may instead be the case that for that stimulus they simply do not perceive a consistent color. Non-synesthetes, when forced to choose colors for letters, are often highly consistent in their responses (Simner et al., 2005), so it is entirely plausible that synesthetes would be highly consistent if forced to choose a color for a grapheme for which they do not consciously perceive a color.

Third, the Eagleman Battery uses an HSL colorpicker, in which the hue slider has two endpoints. This is potentially-problematic for two reasons. First, humans perceive hue in a circular manner: red is similar to magenta, which is similar to purple, and so on. As a result, there may be instances in which a subtle adjustment in hue requires moving the color picker to the complete opposite side, and participants may be less inclined to make adjustments like this when they are effortful. Second, the specific endpoints used by the Eagleman battery are the additive and subtractive primary colors (red, green, blue, yellow, cyan, magenta). It is less difficult and effortful to drag the slider to the edge than it is to choose a precise color off an edge. As a result, the choice of edge colors may confound the distribution of data (especially for control participants), making it appear as if participants choose particularly pure hues much more often than chance.

A final issue with the Eagleman Battery and other online tests is that they are not open source, and thus it was never possible to modify the test-retest experiment to add additional stimuli, translate the experiment to new languages, make modifications to the task, etc. Eagleman et al (2007) did release a similar tool to their battery, the TEXSYN toolkit, but this toolkit was severely limited: it required Matlab and thus could not be administered over the internet. $A$ fully open-source online colorpicker would enable synesthesia researchers to create customized experiments, and to mitigate privacy concerns related to automatic sharing of research data with whichever lab owns the battery.

Here, I test whether potential confounds in the original Eagleman Battery actually influence the data of synesthetes and controls in test-retest experiments. Having determined the optimal set of methodological choices, I next provide open-source code (via Github) to run a test-retest 
consistency experiment using the Qualtrics online platform. Qualtrics is a popular tool for running psychology experiments, used by universities around the world. Qualtrics is GDPR compliant, and many universities have existing site licenses and data processing agreements, enabling hassle-free design and implementation of online experiments. Aside from attribution (users can cite this paper), my code is free to use, modify, and distribute, and enables researchers to easily create their own test-retest consistency tests, with no obligation to share data or authorship.

\section{Methodological Influences on Grapheme-Color Data}

Test-retest-threshold based diagnostic measures of synesthesia rely on the fact that nonsynesthetes have reliably poorer test-retest consistency than synesthetes. However, it is essential to understand the source of this difference in performance, because it has important implications for the external validity of "threshold" measures of synesthesia. For example, if control participants are inattentive or uninvested in the experiment, they may "skip" trials by choosing the initial (random) color of the colorpicker. Because these control subjects are particularly inconsistent, a threshold based on their data might be too liberal a criterion for synesthesia diagnosis, and may misdiagnose attentive non-synesthetes (many of whom will choose some consistent color associations, based on memory or unconscious associations; Simner et al., 2005) as true synesthetes. To determine the potential effect of "skipped" trials on test-retest measures, I collect data from Dutch synesthetes and controls while tracking the (randomly-generated) starting color on each trial.

Participants. Data are from 233 Dutch participants (recruited at the University of Amsterdam and from pre-existing Dutch databases of synesthetes, e.g. from Root et al., 2018), who took a customized test-retest experiment (described in detail in the next section). Particiapnts indicated via subjective report whether or not they experienced grapheme-color synesthesia; 122 participants reported being synesthetes, 56 reported being non-synesthetes, and 55 reported being unsure.

\section{Proportion of skipped trials}

I operationalize a "skipped trial" as a trial in which the color picker was not adjusted - i.e., the final color choice was identical to the randomly-generated initial color. Figure 1 depicts the cumulative distribution function for skipped trials, separated by subjective report of experiencing synesthesia (yes, no, maybe). Subjects who subjectively reported being nonsynesthetes (or of being unsure) frequently skipped trials: approximately $10 \%$ of these subjects skipped more than $30 \%$ of all trials! Because initial color was randomly generated, these trials make non-synesthetes look considerably less consistent than if they are eliminated: the average test-retest consistency (average of the sum of pairwise Euclidean distances for each letter in CIELuv perceptual color space) for non-synesthetes' raw data was an average of 41 CIELuv units worse than test-retest consistency for non-synesthetes' data with skipped trials removed (a highly statistically difference; $t(54)=4.17, \mathrm{p}<0.0005)$. 




Figure 1. Cumulative distribution of skipped trials.

This has real implications for threshold classification measures, especially considering that synesthetes also (occasionally) skipped trials. Replicating the methods of Rothen et al. (2013), we sought to determine the test-retest consistency threshold that best separated synesthetes and non-synesthetes in our sample. With the raw data, our ideal threshold was remarkably similar to Rothen et al's threshold: our ideal threshold was 134.6 (compared to Rothen et al.'s 135.3). After removing skipped trials, our ideal threshold was still remarkably similar (136.9), yet sensitivity was improved $(80.2 \%$ vs. $76.0 \%)$ without a concomitant decrease in specificity (88.5\% for both); in our sample this corresponds to correctly detecting an additional 5 synesthetes with no penalty (no additional misclassification of non-synesthetes as synesthetes or vice versa).

In sum, experiments measuring test-retest consistency in synesthetes should ensure that the (random) initial starting values of the experiment are recorded, so that inattention, lack of motivation/effort, or even accidental misclicks can be partially accounted for. Doing so demonstrably changes the underlying data (making control subjects appear more consistent), and also improves diagnostic accuracy by removing an uninformative signal from the data. Furthermore, detailed measures of subjects' trial performance - for example, mousetracking data of their moment-to-moment manipulation of the colorpicker - could yield even more accurate data, and may also be interesting to analyze in their own right. 


\section{Measuring Test-Retest Consistency using Qualtrics: Code and Instructions}

The test-retest consistency Qualtrics experiment template can be downloaded from the Github repository here: https://github.com/nicholasbroot/synesthesiacolorpicker.

\section{Importing the experiment file(s) into Qualtrics}

There are two separate. QSF experiment files:

1. SynesthesiaColorPicker.qsf contains only the color picker and necessary tracking code, for a single trial. Use this file to create your own experiment from scratch.

2. GCSynTestRetest.qsf is a complete test-retest consistency experiment for graphemecolor associations in English. Use this file if you instead want to modify an existing implementation of a test-retest consistency experiment.

Instructions to import a .QSF survey can be found here:

https://www.qualtrics.com/support/survey-platform/survey-module/survey-tools/import-andexport-surveys/\#ImportingASurvey

\section{Features of the colorpicker}

The colorpicker trial consists of three Qualtrics questions, one displayed and two hidden. This enables data about participants' choices to be recorded in an easily interpretable format:

ColorPick is the main question, in which the color picker is displayed to the participant. The input text box (which is hidden from the participant) is continuously updated with the current color value of the colorpicker, so in the Qualtrics data file, the value of this question will be the final color chosen by the participant. The question is currently written to pull a (text) stimulus from the first column of the settings for Qualtrics' Loop and Merge feature ( $\$ \mid \mathrm{Im}: / /$ Field/1 $\}$ ), which enables different stimuli to be piped in to the same question (e.g., each letter of the alphabet). The code should work "out of the box" for any grapheme stimuli in any language, but it can also be easily modified to present, e.g., sounds instead of letters - just replace the contents of the "stimulus" div (note that it may be necessary to experiment with width and height settings, depending on your stimulus). Do not edit the "rect" and "colorpicker1" divs are unless you are comfortable with CSS/Javascript, as modifying these may break the colorpicker.

ColorPickStartColor is a hidden question used to record the (randomized) starting color of the colorpicker. The starting color is automatically entered into the (hidden) text box. By comparing the value of ColorPick and ColorPickStartColor in the final Qualtrics data file, it is possible to quickly determine whether the participant moved the colorpicker. This data can also be used to, for example, correlate the starting hue with the chosen hue as an additional diagnostic tool for synesthesia (because the starting hue is random, in a true synesthete these values should be uncorrelated). This question should not be modified. 
ColorPickMouseTrack is a hidden question used to record the evolution of the participant's color choice over time. Each time the chosen color of the colorpicker changes (potentially hundreds of times, as a subject drags their mouse around the picker), the newly chosen color along with a timestamp is automatically added to the text box (in the format "time1,color1;time2,color2;..."). This question should not be modified.

\section{Future Modifications of the Colorpicker}

Below, I describe several additions to the colorpicker that are works in progress. The github repository will be updated as these modifications are completed.

\section{Multicolored colorpicker}

Synesthetes sometimes associate more than one color with a grapheme. We recently (Root \& Rouw, In Review) classified the different multicolored experiences of synesthetes into several categories: change over time, piecewise coloring, and "layers" of color. Although synesthetic associations that change over time can be measured with the colorpicker as is (e.g., by creating an extra loop for each additional color that the subject reports), the colorpicker cannot be used to accurately describe these additional multicolored experiences. One solution to this issue is to create a colorpicker that allows participants to "paint" colors onto the letter.

\section{Colorpicker for texture}

Synesthetes frequently describe synesthetic experiences as having "texture" rather than just color. In the future, a colorpicker should enable synesthetes to accurately describe these experiences. However, it is first necessary to determine the range and consistency of these experiences, currently an open question in the literature. Pilot experiments in synesthetes should determine a testable "taxonomy" of texture experiences, so that these textures can be incorporated into a colorpicker for future studies.

\section{Mobile Version}

Although the colorpicker does work on mobile devices, it is unwieldy and I recommend that it only be used on a computer monitor (in my own experiments, I use Qualtrics' "Device Type" condition to prevent the experiment from running on mobile devices). In the future, the colorpicker could be modified to be more mobile friendly, perhaps by making the display size dynamic, so that it adapts to the participant's screen. This comes with quite a set of potential confounds (e.g., accuracy will vary between computer and mobile, between mobile screens of different sizes, etc.), but as there are many potential participants (particularly in non-Western countries) who own a mobile device but not a computer, it is worth considering how to control as best as possible for these confounds to develop a mobile version of the toolkit. 


\section{References}

Asher, J. E., Aitken, M. R., Farooqi, N., Kurmani, S., \& Baron-Cohen, S. (2006). Diagnosing and phenotyping visual synaesthesia: a preliminary evaluation of the revised test of genuineness (TOG-R). Cortex, 42(2), 137-146.

Blake, R., Palmeri, T. J., Marois, R., \& Kim, C. Y. (2005). On the perceptual reality of synesthetic color. Synesthesia: Perspectives from cognitive neuroscience, 47-73.

Eagleman, D. M., Kagan, A. D., Nelson, S. S., Sagaram, D., \& Sarma, A. K. (2007). A standardized test battery for the study of synesthesia. Journal of neuroscience methods, 159(1), 139-145.

Kadosh, R. C., Sagiv, N., Linden, D. E., Robertson, L. C., Elinger, G., \& Henik, A. (2005). When blue is larger than red: Colors influence numerical cognition in synesthesia. Journal of cognitive neuroscience, 17(11), 1766-1773.

Root, N. B., Rouw, R., Asano, M., Kim, C. Y., Melero, H., Yokosawa, K., \& Ramachandran, V. S. (2018). Why is the synesthete's " $A$ " red? Using a five-language dataset to disentangle the effects of shape, sound, semantics, and ordinality on inducer-concurrent relationships in grapheme-color synesthesia. cortex, 99, 375-389.

Simner, J., Ward, J., Lanz, M., Jansari, A., Noonan, K., Glover, L., \& Oakley, D. A. (2005). Nonrandom associations of graphemes to colours in synaesthetic and non-synaesthetic populations. Cognitive neuropsychology, 22(8), 1069-1085.

Simner, J., Mulvenna, C., Sagiv, N., Tsakanikos, E., Witherby, S. A., Fraser, C., ... \& Ward, J. (2006). Synaesthesia: The prevalence of atypical cross-modal experiences. Perception, 35(8), 1024-1033.

Simner, J. (2013). The "rules" of synesthesia. The Oxford Handbook of Synesthesia, 149-164. 